\title{
Spaceborne Synthetic Aperture Radar (SAR) Systems: State of the Art and Future Developments
}

\author{
Alberto Moreira and Gerhard Krieger \\ German Aerospace Center (DLR) \\ Microwaves and Radar Institute, D-82230 Wessling, Germany \\ Email: alberto.moreira@dlr.de
}

\begin{abstract}
This paper first summarizes the state of the art in spaceborne SAR systems and applications. The second part of this paper gives an overview of new concepts, techniques and technologies for future SAR systems, allowing an increase of flexibility in the SAR operation mode as well as a reduction in the overall system costs. Several innovative concepts and technologies as biand multi-static configurations, parasitic SAR, sparse aperture systems and digital beamforming will play an important role for future spaceborne SAR constellations.
\end{abstract}

\section{INTRODUCTION}

Synthetic Aperture Radar (SAR) is a powerful and well established microwave remote sensing technique which enables high resolution measurements of the Earth surface independent of weather conditions and sunlight illumination [1]. The radar system is coherent and transmits frequency modulated, high energy pulses (chirps) at a high pulse repetition frequency (PRF), typically greater than $1 \mathrm{kHz}$ for space-borne systems. The direction of energy propagation is called range while the flight direction is defined as azimuth. The backscattered echoes are received, down converted and stored after analog/digital-conversion. Due to the use of a small antenna length in azimuth, every point on the ground is illuminated by thousands of pulses (Fig. 1). The basic SAR image formation consists of a compression of the chirp signal followed by the azimuth processing which corresponds to the formation of a long synthetic aperture. This is possible since the SAR system preserves the phase information and all received echoes in azimuth can be coherently combined after a proper phase correction. Since the length of the synthetic aperture increases with the range distance, the resulting azimuth resolution becomes independent on the range distance. The theoretical value for the azimuth resolution is equal to the half of the antenna length in azimuth. This allows a high resolution imaging for air- and space-borne SAR systems. Typical azimuth antenna sizes for spaceborne SAR systems in civil applications are from 5 to 14 meters.

Since the first spaceborne SAR system has been launched in 1978, a tremendous technological and application related development has been achieved. Spaceborne SAR systems represent today one of the most demanding remote sensing imaging instruments in a technological sense and this poses a great challenge for the development of new concepts, techniques and technologies for future SAR systems.

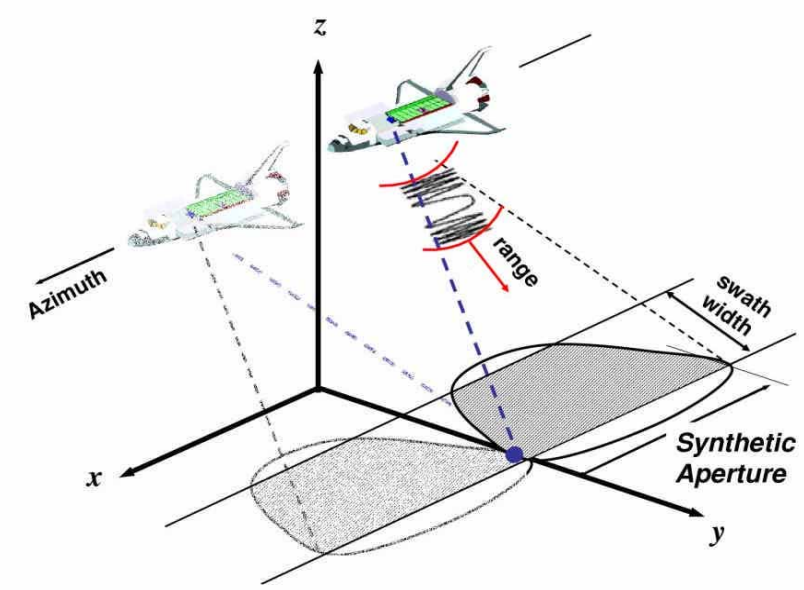

Fig. 1 - Basic geometry for a Synthetic Aperture Radar (SAR) system.

\section{STATE OF THE ART}

In February 2001, the ENVISAT satellite from the European Space Agency (ESA) has been successfully launched. ENVISAT has been developed for environmental monitoring of the Earth atmosphere, ocean and land surfaces and consists of 10 remote sensing instruments. The most complex instrument is the Advanced SAR (ASAR) system which has a phased array antenna with 320 Transmit and Receive (T/R) modules distributed over 32 rows and 10 columns. The T/Rmodules are the most demanding technological development for this SAR system and offer a great flexibility in the selection of the incidence angle range to be imaged as well as the operation of the SAR system in advanced imaging modes as ScanSAR.

ScanSAR imaging mode - In the conventional SAR imaging (stripmap mode), the swath width is limited by the constraint that it is not possible to transmit and receive at the same time. This constraint can be circumvented by using a scanning strategy of the antenna in the elevation by means of an electronic steering [2]. The elevation pattern of the antenna is scanned between several incidence angles to obtain a wider swath composed of several sub-swaths. This imaging mode is called ScanSAR mode (Fig. 2). The synthetic aperture is shared between the sub-apertures, leading to a decreased azimuth resolution. Typical swath width for the ScanSAR mode is between $100 \mathrm{~km}$ and 450 $\mathrm{km}$.

Spotlight imaging mode - Using T/R modules, it is also possible to perform an electronic steering in the azimuth direction leading to the so-called Spotlight 

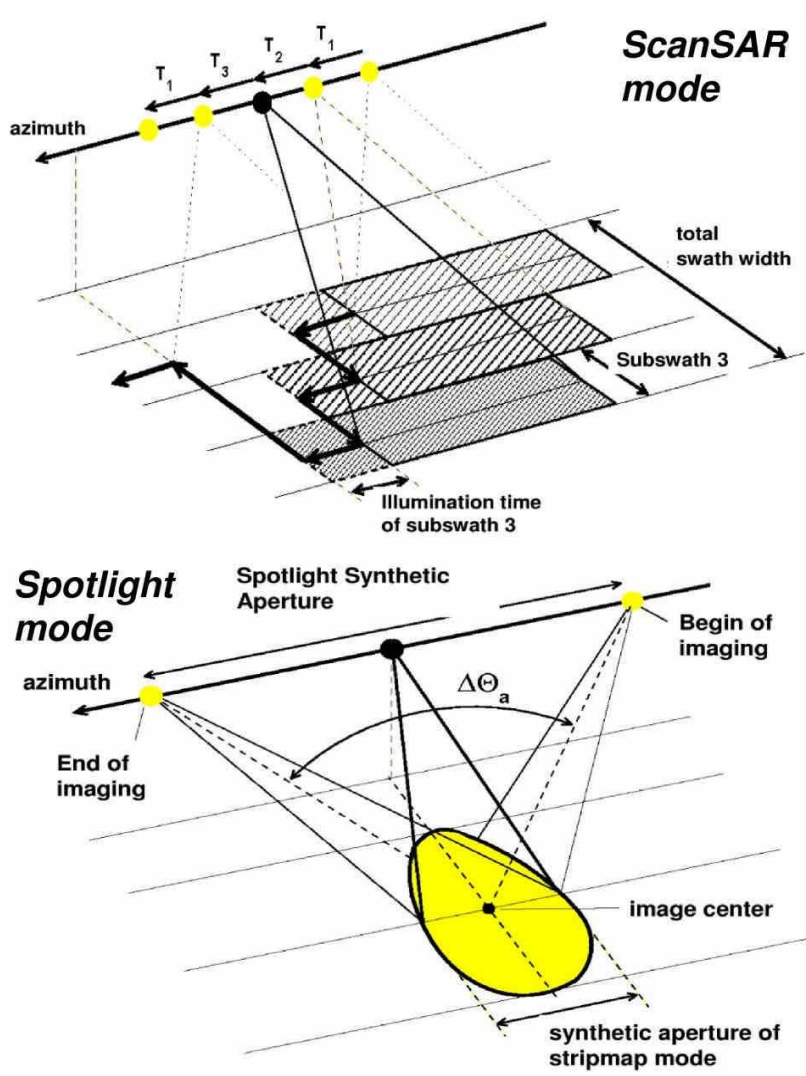

Fig. 2 - Advanced SAR imaging modes

imaging mode [3]. This mode aims to achieve a very high azimuth resolution by increasing the azimuth illumination time (Fig. 2). However, the spotlight mode does not lead to a continuous imaging in the azimuth direction and is mainly used to map small areas with very high resolution. The German satellite TerraSAR-X - having a launch planed for 2005 - will be able to operate in stripmap, ScanSAR and Spotlight modes. In the Spotlight mode, the geometric resolution can be as high as $1 \mathrm{~m} \times 1,5 \mathrm{~m}$ for an image size of ca. 5 x $10 \mathrm{~km}$. TerraSAR-X is being developed in the scope of a Public Private Partnership (PPP) between DLR and Astrium and will be the first German satellite combining both interests of the scientific community and commercial market.

Interferometry - The monostatic SAR imaging with one antenna does not deliver information about the terrain topography since the radar is measuring time delay in the range direction and does not have accurate information about the elevation angle of the backscattered signal which is related to the terrain height. SAR interferometry allows obtaining accurate information about the terrain topography by using two antennas separated by a baseline in the across-track direction [4]. The range difference between the antennas to each target on the ground can be accurately measured by estimating the interferometric phase, i.e. the phase difference between the images acquired by the two antennas. Having the information of the range difference, the corresponding terrain height for each image position can precisely be estimated. The accuracy of the height estimation in the airborne case is in the range of ca. 0,5 to $5 \mathrm{~m}$ and 5 to $20 \mathrm{~m}$ for space-borne systems.

In February 2000, the Shuttle Radar Topography Mission (SRTM) has been launched for an 11 day mission dedicated to a global topographic measurement of the Earth surface. On-board the Shuttle there were two radar systems operating in X- and C-band. The X-band system mapping ca. $40 \%$ of the Earth land mass with ca. 30 x 30 $\mathrm{m}$ horizontal accuracy and 6 meters height accuracy - has been built by DLR in cooperation with the Italian Space Agency (ASI) while the C-Band System - mapping ca. 80 $\%$ of the Earth land area with an horizontal accuracy of $100 \times 100 \mathrm{~m}$ and an height accuracy of ca. $12 \mathrm{~m}$ - was developed by NASA/JPL. The two radar systems were installed in the cargo bay of the Shuttle, while the interferometric antennas were mounted at the end of a 60 $\mathrm{m}$ deployable boom (Fig. 3). The results of the interferometric image processing show that the specified height accuracy can be achieved after a complex calibration procedure, allowing the generation of a global and homogeneous digital elevation model (DEM) of the Earth surface with an unprecedented accuracy.

Use of SAR data - The application fields of SAR data are very wide, many of them being not yet operational and require further research studies and data acquisition campaigns [5]. Examples of application fields are oceanography (wave spectra, wind speed, velocity of ocean currents), glaciology (snow wetness, snow water equivalent, glacier monitoring), agriculture (crop classification and monitoring, soil moisture), geology (terrain discrimination, subsurface imaging), forestry (forest height, biomass, deforestation), deformation monitoring (volcano, earthquake and subsidence monitoring with differential interferometry), environment monitoring (oil spills, flooding, urban growth, global change), cartography and infrastructure planning as well as military surveillance and reconnaissance (strategic policy, tactical assessment).

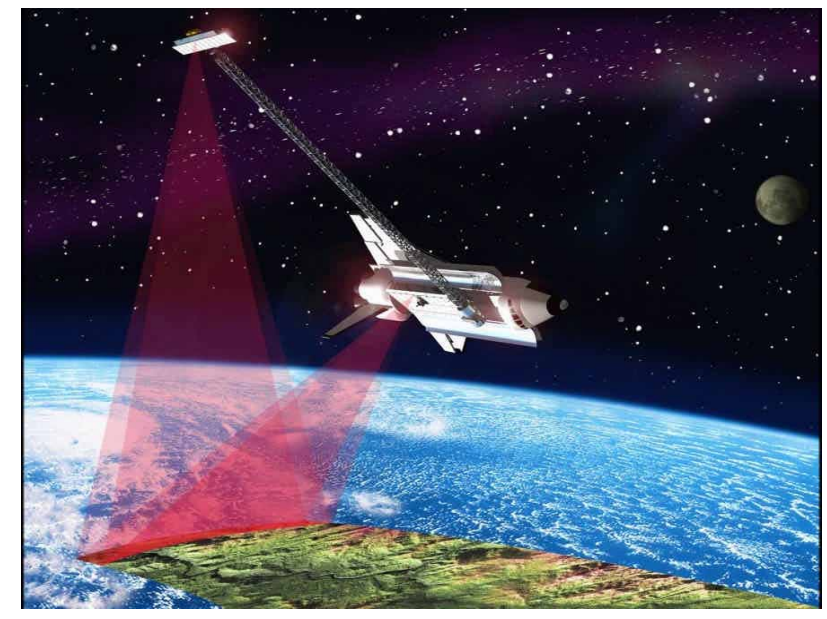

Fig. 3 - Shuttle Radar Topographic Mission (SRTM)

\section{BI- AND MULTISTATIC SAR}

Bi- and multistatic SAR operates with distinct transmit and receive antennas which are mounted on separate platforms. Such a spatial separation has several operational advantages which will increase the capability, reliability and flexibility of future SAR missions. Important additional features provided by multistatic constellations and clusters of co-operating SAR satellites can be summarized as follows: 


\section{Interferometric Cartwheel}

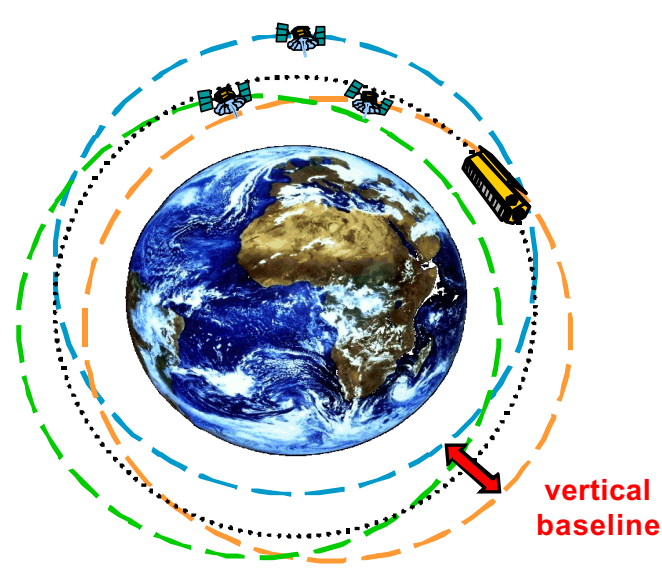

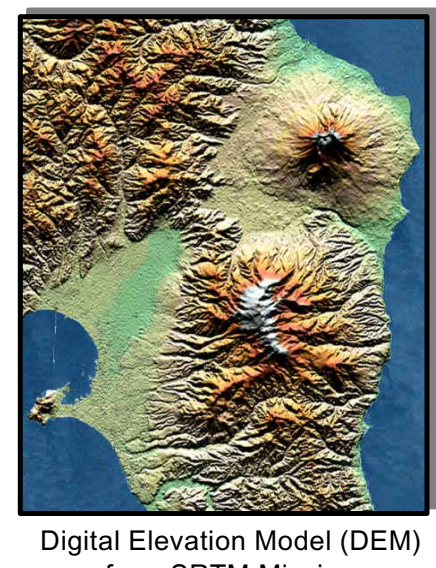

from SRTM Mission

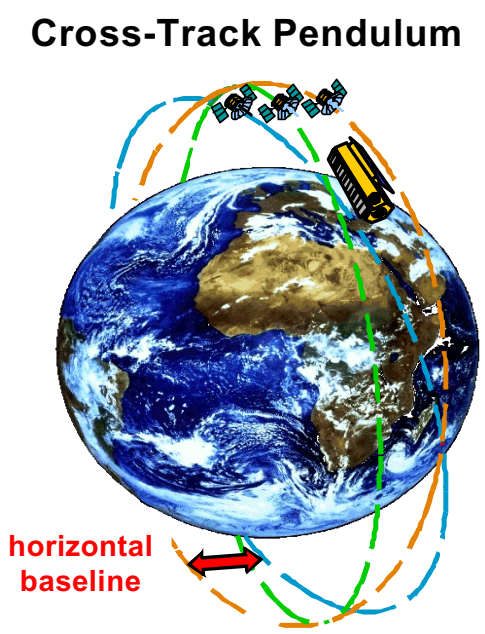

Fig. 4. Interferometric SAR constellation for high resolution topographic mapping of the Earth consisting of a master satellite and 3 receiver satellites. Left: Receiver satellites with a slightly eccentric orbit having different vertical shifts for each orbit position (Cartwheel, [6]). Right: Receiver satellites with circular orbits and a horizontal displacement (Pendulum, [7]). Both constellations are suitable for global mapping of the Earth topography with an unprecedented height accuracy of ca. 2 meters [7].

Single-pass interferometry and sparse aperture sensing: $\mathrm{Bi}-$ and multistatic $\mathrm{SAR}$ constellations offer a natural way to implement single-pass interferometry in space. The use of receivers on different platforms allows for very large baselines, thereby increasing significantly the interferometric performance in comparison to systems relying on a single platform like SRTM. A cost efficient implementation of such systems can be achieved by the combination of a conventional monostatic SAR satellite with one or more passive receivers (Fig. 4). Such systems may easily be upgraded for innovative applications like sparse aperture sensing for enhanced velocity estimation and traffic monitoring, precise localization, polarimetric interferometry for the quantitative extraction of a broad range of geo- and biophysical parameters, or even tomography which enables a real 3-D imaging of the vegetation structure for biomass estimation on a global scale.

Frequent monitoring and increased coverage: Most users require instant access to up-to-date SAR data. The revisit times of current spaceborne SAR sensors - ranging from several days to several weeks - will not suffice for important applications like traffic monitoring, risk and disaster management, or security. Distributed constellations with multiple SAR satellites have the potential to shorten the revisit times substantially. One affordable approach is the use of multiple passive receiver satellites in conjunction with a geostationary illuminator. This concept allows for a systematic reduction of the revisit times as well as an upgrade to other imaging modes by increasing the number of low-cost receivers. Multiple missions may also share a common illuminator, thereby decreasing the costs of each individual mission significantly. A different approach is the use of satellites in geosynchronous or medium earth orbits. Major advantages of such a concept are, besides the short revisit times, the broad coverage and the huge simultaneous access area.

Multi-angle observation and flexible geometry: The use of multiple platforms enables a flexible and reconfigurable imaging geometry. By this, one may gain important additional information about natural and manmade targets. An evaluation of the bistatic radar cross section will improve target classification and it is expected that the acquisition of bistatic SAR images from multiple angles will be of fundamental importance to many branches of Earth science. Multi-angle observations allow also for an improved imaging performance due to reduced retro-reflector effects or forward scattering, and the simultaneous signal reception by multiple receivers may increase the SNR, thereby lowering the power requirements of the transmitter. $\mathrm{Bi}$ - and multistatic satellite formations can also be optimized for applications like super-resolution, ambiguity suppression, or high resolution wide swath imaging.

Cost reduction: The distributed functionality in bi- and multistatic SAR allows for a natural separation of the radar payloads and will therefore strongly support the use of small, low-cost satellites in the future. An example is a multistatic SAR with multiple passive receivers. The reduced power demands and the possible use of deployable antennas allow for an accommodation of the receiver payload on micro-satellites. Small, multi-purpose satellites will also support a modular design and the reuse of major building blocks shortens development time, increases flexibility, and reduces costs. All this will enable affordable and powerful SAR missions with a broad spectrum of remote sensing applications.

Challenges: Multi-static constellations require precise phase synchronization between the radar systems to allow a coherent processing for the combined image formation. This can be achieved either by means of ultra-stable oscillators or microwave links. Additional requirements are the accurate knowledge of the relative orbit trajectory and positioning, satellite formation flight, lightweight antenna technology and multi-static image formation algorithms.

\section{DIGITAL BEAMFORMING}

A further very promising technique for future SAR systems is digital beamforming on receive where the antenna is split into multiple sub-apertures with 


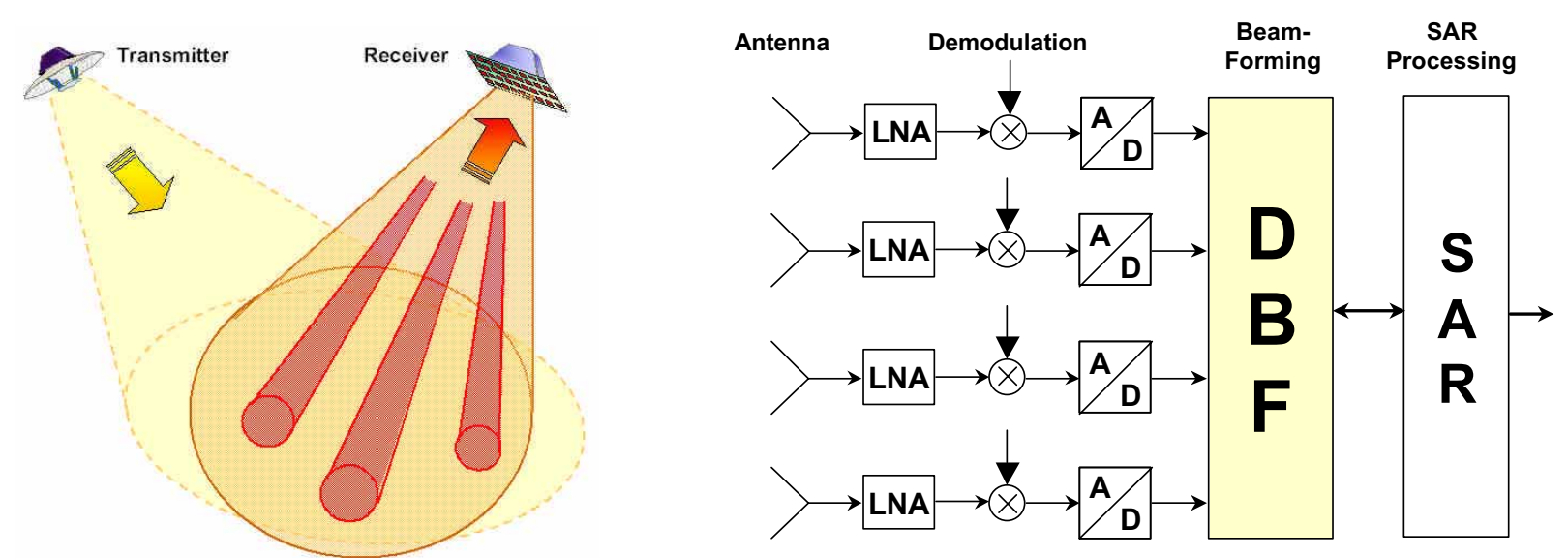

Fig. 5. Bistatic SAR with digital beamforming on receive. Left: Illumination of a large footprint and reception of the scattered signals with multiple beams. Right: Block diagram of digital beamforming on receive (see text).

individual receiver channels. As shown in Figure 5 on the right, each sub-aperture signal is individually amplified, down converted, and digitized. The digital signals are then combined in a dedicated signal processor to form multiple antenna beams with arbitrary shapes. Since the beamforming can be implemented in a programmable computer, it will become easy to reconfigure the radar for different imaging modes. This leads to a high flexibility in operating future SAR systems. Furthermore, the opportunity to gather additional information about the direction of the scattered radar echoes a posteriori to signal reception will enhance the performance and enable a wealth of new applications. Examples are multiple baseline along-track interferometry for traffic monitoring, the dynamic suppression of ambiguities and/or interferences, or the optimization of the antenna gain across the image swath.

Digital beamforming is also very promising in combination with bistatic SAR. One example is the use of a dedicated transmitter for the illumination of a wide image swath (cf. Fig. 5, left). The directional information acquired by digital beamforming on receive can then be used to separate simultaneously recorded signals from multiple spots or sub-swaths. By forming multiple antenna beams in azimuth, it becomes possible to enhance the along-track resolution without the necessity to increase the pulse repetition frequency. Ambiguities in the receiver signal can be suppressed by an appropriate nullsteering of the antenna pattern in range and azimuth. This technique enables the mapping of large areas with very compact SAR sensors. Digital beamforming on receive can also be used to improve both the signal-to-noise ratio and the radiometric resolution of processed SAR images. Furthermore, an appropriate sub-sampling and/or time variant bit allocation will allow for a reduction of the high data rate after the $\mathrm{A} / \mathrm{D}$ conversion while preserving the spatial information of the wave-front impinging on the antenna array. Such an approach is especially promising in combination with transmitters that use frequency modulated continuous wave (FMCW) illumination [8].

The technique of digital beamforming can further be extended to multi-static SAR constellations with several passive receivers. The coherent combination of multiple receiver signals can then be treated in the framework of digital beamforming with sparse antenna arrays. The opportunity to form very narrow beams will, e.g., allow for a space variant suppression of range and azimuth ambiguities. This will in turn lead to a reduction of the required antenna size, thereby enabling cost-effective and powerful SAR missions with reconfigurable satellite arrays. Such constellations may serve a broad range of different applications by integrating interferometry, tomography, super-resolution, and wide swath imaging in one remote sensing mission.

\section{CONCLUSION}

A major requirement for many applications as hazard monitoring is a revisit time smaller than one day (even below one hour for traffic monitoring). Present SAR systems consisting of one satellite achieve a revisit time of ca. one month. Constellations of small satellites will fulfill the requirement posed by these applications. The aforementioned technologies and concepts (digital beamforming, small receiver satellites and multi-static configurations) will play an important role in the future SAR development towards the formation of satellite constellations. In addition, the merge of remote sensing, navigation and communication applications will allow each user, in any place, at the right time to access the required information.

\section{REFERENCES}

[1] Curlander, J., McDonough, R., Synthetic Aperture Radar: Systems and Signal Processing. New York: Wiley, 1991.

[2] Tomiyasu, K., Conceptual Performance of a Satellite Borne, Wide Swath Synthetic Aperture Radar. IEEE Trans. Geoscience and Remote Sensing, 19(2), 1981, pp. 108-116.

[3] Carrara, W. et al, Spotlight Synthetic Aperture Radar: Signal Processing Algorithms. Boston: Artech, 1995.

[4] Bamler, R. and Hartl, P., Synthetic Aperture Radar Interferometry, Inverse Problems. vol. 14, 1998, pp. 1-54.

[5] Henderson, F. und Lewis, A., Manual of Remote Sensing. Principles and Applications of Imaging Radar. New York: John Wiley \& Sons, 1998.

[6] Massonnet, D., Capabilities and Limitations of the Interferometric Cartwheel, IEEE Trans. Geosci. Remote Sens., 39(3), 2001, pp. 506-520.

[7] Krieger, G., H. Fiedler, J. Mittermayer, K. Papathanassiou, A. Moreira, Analysis of Multistatic Configurations for Spaceborne SAR Interferometry, IEE Proc. Radar, Sonar, Navig., 150(3), 2003, pp. 87-96.

[8] Krieger, G. and A. Moreira, Potentials of Digital Beamforming in Bi- and Multistatic SAR, Proc. IGARSS, Toulouse, 2003. 